\title{
A Perspective on the Application of Pro-/Synbiotics in Clinical Practice
}

\author{
Xiang-Dong Wu ${ }^{1,2}$, Yu Chen ${ }^{1}$ and Wei Huang ${ }^{1 *}$ \\ ${ }^{1}$ Department of Orthopaedic Surgery, The First Affiliated Hospital of Chongqing Medical University, Chongqing, China, \\ ${ }^{2}$ Evidence-Based Perioperative Medicine 07 Collaboration Group, Hong Kong, Hong Kong
}

Keywords: probiotics, prebiotics, synbiotics, microbiota, clinical practice

Our previous meta-analysis (Wu et al., 2017) evaluated broadly the available evidence and confirmed that pro-/synbiotics supplementation is effective in preventing or controlling the incidence of surgical site infections (SSIs) after a surgical procedure, yet subgroup analyses indicated the primary outcome was influenced by various factors (Figure 1), which might affect the robustness of the conclusion and further confuse the clinical practice. Lack of proper application of pro-/synbiotics is the primary cause of ineffective management. Thus, several issues around the practicalities of pro-/synbiotics should be taken into account.

\section{WHAT ARE THE BEST REGIMENS OR CONSTITUTIONS?}

Theoretically, prebiotics could reach the colon intact and selectively stimulate the growth and activities of probiotics, and synbiotics combining probiotics with prebiotics should work better than probiotics alone (Gibson and Roberfroid, 1995). However, whether synbiotics therapy provides

\section{OPEN ACCESS}

Edited by:

Aldo Corsetti,

University of Teramo, Italy

Reviewed by:

Giuseppe Spano, University of Foggia, Italy

Giovanna Suzzi,

University of Teramo, Italy

*Correspondence:

Wei Huang

drhuangwei68@gmail.com

Specialty section:

This article was submitted to

Food Microbiology,

a section of the journal

Frontiers in Microbiology

Received: 05 March 2017 Accepted: 28 April 2017 Published: 23 May 2017

Citation:

Wu X-D, Chen Y and Huang W (2017) A Perspective on the Application of Pro-/Synbiotics in Clinical Practice. Front. Microbiol. 8:866. doi: 10.3389/fmicb.2017.00866 greater benefit than either the probiotics or prebiotics on its own still need further directly head-to-head comparison (Tang and Lodge, 2016).

We also detected multi-strains are more effective than single-strain, this might attribute to a synergic effect (Timmerman et al., 2004). But the subsequent issue is all pro-/synbiotics often get lumped together, which consist of many different species and concentrations vary wildly between products. As such lumping studies together unselectively is prone to lead to conflicting results. Therefore, further trials applying slightly more selective probiotics in homogenous population would conduce to show a bit more consistent results.

Previous studies have established that individual probiotics can have distinct strain-specific effects (Luyer et al., 2005; Kekkonen et al., 2008; Tang et al., 2010; Frei et al., 2015). Similarly, different prebiotic oligosaccharides have different microbiota-modifying and immunomodulatory properties (Lee and Salminen, 2009). Hence, investigation of individual probiotics and prebiotics effects is conducive to designing and using combined regimens for specific clinical conditions. Another weak point of many studies done in the field of pro-/synbiotics is the carrier, which may partially influence the effectiveness (Moradi et al., 2013; Mohammadmoradi et al., 2015).

One more major problem is the usage of wrong strains, some reported probiotics bacteria are even among known pathogens (Boyle et al., 2006; Hempel et al., 2011). Microbiologists have explained that the behavior of a microbe depends on several factors, we are ignoring the warnings (Sanders et al., 2010; Pirofski and Casadevall, 2012; Didari et al., 2014; Doron and Snydman, 2015). In vitro and in vivo tests on a range of probiotics cultures for their ability to inhibit a panel of pathogens are desperately needed to establish an effective routine (Papadimitriou et al., 2015). It should be further emphasized that strains used in multi-strains and multi-species of probiotics and prebiotics should be compatible or, preferably, synergistic (Timmerman et al., 2004). Overview, emerging reports manifest that we should choose the right strains alongside with proper prebiotics and adequate carrier to reach a synergic effect. But the best regimens or constitutions still need copious further studies. 


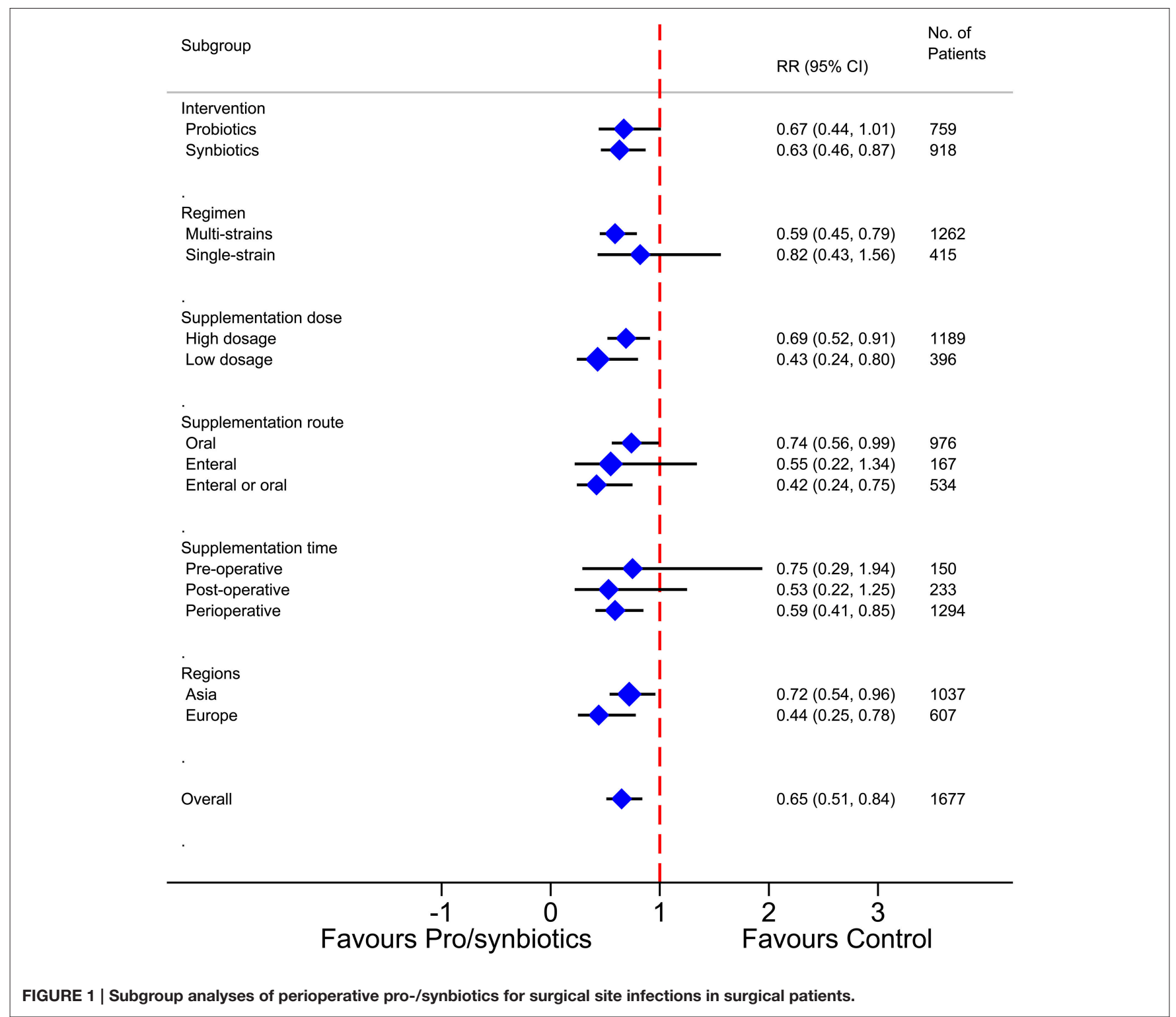

\section{HOW TO USE THE REGIMEN?}

It should be a state-of-the-art technique to use pro-/synbiotics regimen to modulate the gastrointestinal microbiota. But the key question is we know very little about how to use the regimen appropriately.

Subgroup analysis favors high does rather than low does of pro-/synbiotics. It may be mainly due to that the human microbiota contains as many as $10^{14}$ bacterial cells, and majority reside in our colon where densities approach $10^{11} \sim 10^{12}$ cells/g, which is the highest record for any microbial habitat bacterial cells (Savage, 1977; Whitman et al., 1998; Ley et al., 2006). Thus only high does more than $10^{10} \mathrm{CFUs}$ /day can reach and colonize the gut to further inducing changes in the colorectal microbiota and stabilize microbial communities (Hemarajata and Versalovic, 2013). And studies with inadequate dose of strains didn't experience any improvements. Nevertheless, one dose level cannot be assumed to be always effective for all strains (Sanders, 2008).

The mode of administration is rather important, because pro-/synbiotics are fragile and can be killed easily by heat or stomach acid (Alvarez-Calatayud and Margolles, 2016). This explains why enteral/oral route is more effective than oral and enteral. It is still difficult to measure potential probiotics that survival and colonization to the gut wall. The impact of product format on pro-/synbiotics function has yet to be explored in depth.

The timing for the colonization and proliferation of the gut by the probiotics is also important. We found peri-operative administration is more effective than pre-operative or postoperative administration, this may mainly due to the slow rate of cell division (Lee et al., 2004). Thus longer administration would 
accelerate the accumulation of microbes, and altered which into a healthy microbiota.

One more interesting issue is the regional difference. Subgroup analysis indicated pro-/synbiotics are more effective in Europe than Asia. We all know that the food habit and environmental condition change the microflora, which would induce intestinal microbiota difference in distinct areas (Marathe et al., 2012). Therefore, we should also take regional specificity into consideration when developing the best regimens.

The statement that pro-/synbiotics "improve the balance of microflora" is often declared by the producers. Although our understanding of the composition and functions of the gut microbiota has increased exponentially during the past decade (Arumugam et al., 2011), we still don't know exactly what roles most of the intestinal bacteria are playing, and how they are interacting with each other and the hosts. Gastrointestinal tract remains a challenging environment to explore, sample, and to describe (Marchesi et al., 2016). Dose colonize, proliferate, and alter the population corresponding to an "improved balance"? Without more knowledge of the larger percentage of unknown microbiota, we cannot learn about whether or not the gut microbiota is a potential therapeutic target which we can modulate in order to treat or prevent specific diseases (Marchesi et al., 2016).

Although there is evidence to support pro-/synbiotics use in reducing SSIs for patients undergoing a surgical procedure, strong scientific evidence to support specific uses of pro/synbiotics for most health conditions is lacking. Therefore, the U.S. Food and Drug Administration (FDA) has not approved any pro-/synbiotics for preventing or treating any health problem. Absence of a legal definition allows many pro-/synbiotics are sold as dietary supplements on various levels of quality. The cost of these products can be substantial and may not be covered under patients' health care plan (Matarese et al., 2003; Visich, 2010).

\section{CALL FOR FURTHER STUDIES}

Considering the current encouraging evidence and challenges, more research in humans to further document the health benefits of pro-/synbiotics as adjunct therapy are needed (Schaeffer, 2017). First, well-designed and properly powered trials with appropriately chosen of strains should be performed, current literature reported conflicting observations may partly be due to poor study design and poor choice of strain (Marchesi et al., 2016). Next, further studies are needed to explore the strain specificity, does specificity, strain combinations characterized for the specific health target, and ultimately achieve using predefined administration mode of specific pro-/synbiotics regimen for definite disease in certain population or region. Third, the effect of product format on pro-/synbiotics function also needs to be explored in depth. Apart from viable bacterial density, other factors like pro-/synbiotics growth during manufacture, enteric coating, preservation technology, metabolic state, and combination with other functional ingredients, may also play a role in the effectiveness of a product (Sanders, 2008). Finally, more research is warranted to understand the human microbiota, there is also a persistent lack of understanding as to the very nature of pro-/synbiotics. We could only speculate that pro/synbiotics may actually facilitate a return to normal balance status after a perturbation of the microbiota (e.g., because of the use of antibiotics, traditional mechanical bowel preparation or surgical stress) or may reduce the degree of change invoked by such challenges (Sanders, 2008). Therefore, better understanding the mechanisms of the pro-/synbiotics interactions with microbiota would contribute to elucidating how these benefits are achieved, as well as developing novel therapeutics and strategies to modulate the microbiota (Ford et al., 2014).

\section{LIMITATIONS}

Our opinion has limitations. First, the above mentioned clinical application perspective was based on the results of six subgroup analyses, and the results of the subgroup analyses should not be interpreted as definitive conclusions since they are observational by nature and are not based on randomized comparisons (Sun et al., 2010). Furthermore, the results of test of interaction suggest no significant differences between subgroups; all subgroup analyses were not specified a priori but post hoc analyses, the numbers of studies in the "significant subgroups" are occasionally small, and it is more likely to overestimated the intervention effect compared with larger sample size (Kjaergard et al., 2001; Sterne and Egger, 2001). Therefore, these subgroup analyses results should be interpreted with caution as we might not have had enough power to detect a difference. Next, the included studies were methodologically and biologically heterogeneous, which mainly reflected in the huge variability in clinical settings, pro-/synbiotics strains, routine supplementation does, administration route, control intervention, and stringency of trial execution. These varieties induced equivocal results and further limited the validity and generalizability of our findings. However, our perspective still pointed out the shortcomings of the current research field, and strengthened the keystone for further clinical practice that were worth investigating or revisiting. Lastly, further trials should pay additional concern to the conflicts of interest. Few included studies mentioned industry funding, and small prospective studies sponsored by the pro-/synbiotics industry are likely to be biased (Bekelman et al., 2003; Bero, 2013).

Currently, it is hard to give an exhaustive advice or elaborate guidance on pro-/synbiotics application in clinical practice regarding preliminary findings. To some extent, synbiotics combined multiple-strains probiotics with prebiotics, administrated perioperatively at a high dose should be more effective. To play the greatest degree of pro-/synbiotics in clinic, considerable amount of in vitro work are warrant, and intensive in vivo exploration followed by randomized, double blind, placebo controlled clinical trials need to be performed.

\section{AUTHOR CONTRIBUTIONS}

XW: Contributed substantially to conception and design, acquisition of data, analysis and interpretation of data; drafted the article; gave final approval of the version to be published; 
agreed to act as guarantor of the work. YC: Contributed substantially to acquisition of data, analysis and interpretation of data; drafted the article; gave final approval of the version to be published; agreed to act as guarantor of the work. WH:
Contributed substantially to conception and design, acquisition of data, analysis and interpretation of data; revised it critically for important intellectual content; gave final approval of the version to be published; agreed to act as guarantor of the work.

\section{REFERENCES}

Alvarez-Calatayud, G., and Margolles, A. (2016). Dual-coated lactic acid bacteria: an emerging innovative technology in the field of probiotics. Future Microbiol. 11, 467-475. doi: 10.2217/fmb.15.150

Arumugam, M., Raes, J., Pelletier, E., Le Paslier, D., Yamada, T., Mende, D. R., et al. (2011). Enterotypes of the human gut microbiome. Nature 473, 174-180. doi: 10.1038/nature09944

Bekelman, J. E., Li, Y., and Gross, C. P. (2003). Scope and impact of financial conflicts of interest in biomedical research: a systematic review. JAMA 289, 454-465. doi: 10.1001/jama.289.4.454

Bero, L. (2013). Industry sponsorship and research outcome: a Cochrane review. JAMA Intern. Med. 173, 580-581. doi: 10.1001/jamainternmed.2013.4190

Boyle, R. J., Robins-Browne, R. M., and Tang, M. L. (2006). Probiotic use in clinical practice: what are the risks? Am. J. Clin. Nutr. 83, 1256-1264; quiz 1446-1257.

Didari, T., Solki, S., Mozaffari, S., Nikfar, S., and Abdollahi, M. (2014). A systematic review of the safety of probiotics. Expert Opin. Drug Saf. 13, 227-239. doi: $10.1517 / 14740338.2014 .872627$

Doron, S., and Snydman, D. R. (2015). Risk and safety of probiotics. Clin. Infect. Dis. 60(Suppl. 2), S129-S134. doi: 10.1093/cid/civ085

Ford, A. C., Quigley, E. M., Lacy, B. E., Lembo, A. J., Saito, Y. A., Schiller, L. R., et al. (2014). Efficacy of prebiotics, probiotics, and synbiotics in irritable bowel syndrome and chronic idiopathic constipation: systematic review and meta-analysis. Am. J. Gastroenterol. 109, 1547-1561. doi: 10.1038/ajg.2014.202

Frei, R., Akdis, M., and O’Mahony, L. (2015). Prebiotics, probiotics, synbiotics, and the immune system: experimental data and clinical evidence. Curr. Opin. Gastroenterol. 31, 153-158. doi: 10.1097/MOG.0000000000000151

Gibson, G. R., and Roberfroid, M. B. (1995). Dietary modulation of the human colonic microbiota: introducing the concept of prebiotics. J. Nutr. 125, 1401-1412.

Hemarajata, P., and Versalovic, J. (2013). Effects of probiotics on gut microbiota: mechanisms of intestinal immunomodulation and neuromodulation. Therap. Adv. Gastroenterol. 6, 39-51. doi: 10.1177/1756283X12459294

Hempel, S., Newberry, S., Ruelaz, A., Wang, Z., Miles, J. N., Suttorp, M. J., et al. (2011). Safety of probiotics used to reduce risk and prevent or treat disease. Evid. Rep. Technol. Assess. (Full Rep), 1-645.

Kekkonen, R. A., Lummela, N., Karjalainen, H., Latvala, S., Tynkkynen, S., Jarvenpaa, S., et al. (2008). Probiotic intervention has strain-specific antiinflammatory effects in healthy adults. World J. Gastroenterol. 14, 2029-2036. doi: 10.3748/wjg.14.2029

Kjaergard, L. L., Villumsen, J., and Gluud, C. (2001). Reported methodologic quality and discrepancies between large and small randomized trials in meta-analyses. Ann. Intern. Med. 135, 982-989. doi: 10.7326/0003-4819-135-11-200112040-00010

Lee, Y., Ho, P., Low, C., Arvilommi, H., and Salminen, S. (2004). Permanent colonization by Lactobacillus casei is hindered by the low rate of cell division in mouse gut. Appl. Environ. Microbiol. 70, 670-674. doi: 10.1128/AEM.70.2.670-674.2004

Lee, Y. K., and Salminen, S. (2009). Handbook of Probiotics and Prebiotics. Hoboken, NJ: John Wiley \& Sons, Inc.

Ley, R. E., Peterson, D. A., and Gordon, J. I. (2006). Ecological and evolutionary forces shaping microbial diversity in the human intestine. Cell 124, 837-848. doi: 10.1016/j.cell.2006.02.017

Luyer, M. D., Buurman, W. A., Hadfoune, M., Speelmans, G., Knol, J., Jacobs, J. A., et al. (2005). Strain-specific effects of probiotics on gut barrier integrity following hemorrhagic shock. Infect. Immun. 73, 3686-3692. doi: 10.1128/IAI.73.6.3686-3692.2005

Marathe, N., Shetty, S., Lanjekar, V., Ranade, D., and Shouche, Y. (2012). Changes in human gut flora with age: an Indian familial study. BMC Microbiol. 12:222. doi: $10.1186 / 1471-2180-12-222$

Marchesi, J. R., Adams, D. H., Fava, F., Hermes, G. D., Hirschfield, G. M., Hold, G., et al. (2016). The gut microbiota and host health: a new clinical frontier. Gut 65, 330-339. doi: 10.1136/gutjnl-2015-309990

Matarese, L. E., Seidner, D. L., and Steiger, E. (2003). The role of probiotics in gastrointestinal disease. Nutr. Clin. Pract. 18, 507-516. doi: $10.1177 / 0115426503018006507$

Mohammadmoradi, S., Javidan, A., Kordi, J., and Goudarzi, M. H. (2015). Comparing the effect of ultra-filtered feta cheese and yoghurt as probiotic carriers on lipid profile: a double blinded randomized controlled trial. Med. J. Nutr. Metab. 8, 27-36. doi: 10.3233/MNM-140026

Moradi, S. M., Javidan, A., and Isfahani, H. N. (2013). Effects of probiotic ultrafiltered feta cheese and raw chicory root extract on lipid profile in healthy adult volunteers: a triple-blinded randomized controlled trial. Med. J. Nutr. Metab. 6, 199-206. doi: 10.1007/s12349-013-0130-6

Papadimitriou, K., Zoumpopoulou, G., Foligné, B., Alexandraki, V., Kazou, M., Pot, B., et al. (2015). Discovering probiotic microorganisms: in vitro, in vivo, genetic and omics approaches. Front. Microbiol. 6:58. doi: $10.3389 /$ fmicb.2015.00058

Pirofski, L. A., and Casadevall, A. (2012). Q and A: what is a pathogen? A question that begs the point. BMC Biol. 10:6. doi: 10.1186/1741-7007-10-6

Sanders, M. E. (2008). Probiotics: definition, sources, selection, and uses. Clin. Infect. Dis. 46(Suppl. 2), S58-S61. discussion: S144-S151. doi: 10.1086/ 523341

Sanders, M. E., Akkermans, L. M., Haller, D., Hammerman, C., Heimbach, J. T., Hörmannsperger, G., et al. (2010). Safety assessment of probiotics for human use. Gut Microbes 1, 164-185. doi: 10.4161/gmic.1.3.12127

Savage, D. C. (1977). Microbial ecology of the gastrointestinal tract. Annu. Rev. Microbiol. 31, 107-133. doi: 10.1146/annurev.mi.31.100177. 000543

Schaeffer, E. M. (2017). Re: effects of perioperative supplementation with pro/synbiotics on clinical outcomes in surgical patients: a meta-analysis with trial sequential analysis of randomized controlled trials. J. Urol. 197, 1274-1275. doi: 10.1016/j.juro.2017.01.016

Sterne, J. A., and Egger, M. (2001). Funnel plots for detecting bias in metaanalysis: guidelines on choice of axis. J. Clin. Epidemiol. 54, 1046-1055. doi: 10.1016/S0895-435600377-8

Sun, X., Briel, M., Walter, S. D., and Guyatt, G. H. (2010). Is a subgroup effect believable? Updating criteria to evaluate the credibility of subgroup analyses. BMJ 340:c117. doi: 10.1136/bmj.c117

Tang, M. L., Lahtinen, S. J., and Boyle, R. J. (2010). Probiotics and prebiotics: clinical effects in allergic disease. Curr. Opin. Pediatr. 22, 626-634. doi: 10.1097/mop.0b013e32833d9728

Tang, M. L., and Lodge, C. J. (2016). Examining the evidence for using synbiotics to treat or prevent atopic dermatitis. JAMA Pediatr. 170, 201-203. doi: 10.1001/jamapediatrics.2015.4406

Timmerman, H. M., Koning, C. J., Mulder, L., Rombouts, F. M., and Beynen, A. C. (2004). Monostrain, multistrain and multispecies probiotics-A comparison of functionality and efficacy. Int. J. Food Microbiol. 96, 219-233. doi: 10.1016/j.ijfoodmicro.2004.05.012

Visich, K. L. (2010). The prophylactic use of probiotics in the prevention of radiation therapy-induced diarrhea. Clin. J. Oncol. Nurs. 14, 467. doi: 10.1188/10.CJON.467-473

Whitman, W. B., Coleman, D. C., and Wiebe, W. J. (1998). Prokaryotes: the unseen majority. Proc. Natl. Acad. Sci. U.S.A. 95, 6578-6583. doi: 10.1073/pnas.95.12.6578

Wu, X. D., Liu, M. M., Liang, X., Hu, N., and Huang, W. (2017). Effects of perioperative supplementation with pro-/synbiotics on clinical outcomes in surgical patients: a meta-analysis with trial sequential analysis of randomized controlled trials. Clin. Nutr. 197, 1274-1275. doi: 10.1016/j.clnu.2016. 10.015 
Conflict of Interest Statement: The authors declare that the research was conducted in the absence of any commercial or financial relationships that could be construed as a potential conflict of interest.

The reviewer GSu and handling Editor declared their shared affiliation, and the handling Editor states that the process nevertheless met the standards of a fair and objective review.
Copyright $\odot 2017 \mathrm{Wu}$, Chen and Huang. This is an open-access article distributed under the terms of the Creative Commons Attribution License (CC BY). The use, distribution or reproduction in other forums is permitted, provided the original author(s) or licensor are credited and that the original publication in this journal is cited, in accordance with accepted academic practice. No use, distribution or reproduction is permitted which does not comply with these terms. 